International Journal of Distributed and Parallel Systems (IJDPS) Vol.3, No.4, July 2012

\title{
Self Configurable Re-link Establishment using Continuous Neighbour Discovery in Asynchronous Wireless Sensor Networks
}

\author{
M.Sreedevi ${ }^{1}$ NADILLA KHADAR VALLI ${ }^{2}$ Dr. R. Seshadri ${ }^{3}$ \\ ${ }^{1}$ Assoc. Prof., C.S.E, MITS, Madanapalle, A.P \\ ${ }^{2}$ M.Tech, MITS, Madanapalle, A.P \\ ${ }^{3}$ Director, Computer Center, S.V.U, Tirupati, A.P
}

\begin{abstract}
In many sensor networks the nodes are static and node connectivity is matter to changes because of disturbance in wireless communication, transmission power varies, or loss of synchronization between neighbouring nodes. Hence, the immediate neighbours is aware of its next sensor, it must continuously maintain its view, we call this process as continuous neighbour discovery. In this work we distinguish between neighbour discovery during sensor network initialization and as well as in it's network life time. We focus on the latter and view it as a joint task of all the nodes in every connected segment. Each sensor employs a simple protocol in a coordinate effort to reduce power consumption without increasing the delay required to detect hidden sensors in the network.
\end{abstract}

\section{KEYWORDS}

Neighbour discovery mechanisms, network life time, delay, static nodes, sensor activity.

\section{INTRODUCTION}

A wireless sensor network (WSN) consists of spatially distributed autonomous sensors to monitor physical or environmental conditions, such as temperature, sound, vibration, pressure, motion or pollutants and to cooperatively pass their data through the network to a main location. The more modern networks are bi-directional, also enabling control of sensor activity. The WSN is built of "nodes" - from a few to several hundreds or even thousands, where each node is connected to one (or sometimes several) sensors. In the sensor network model considered in this paper, the nodes are placed randomly over the area of interest for providing efficient communication each sensor node need to detect their immediate neighbours. A special neighbour discovery scheme should be used when the sensor networks with low and irregular traffic. This paper mainly analyzes the existing neighbour discovery scheme and resources such as energy, memory, computational speed and communications bandwidth of wsn.

In most sensor networks the nodes are static. Nevertheless, node connectivity is subject to changes because of disruptions in wireless communication, transmission power changes, or loss of synchronization between neighbouring nodes

When we compare with traditional wireless communication networks like MANET's and cellular networks the WSN's are having unique characteristics such as high energy computation, huge number of node deployment and some of the storage capability [1],communication i.e. continuous neighbour discovery . Which discussed so many number of new challenging in the communication, development \& deployment of the WSNs? In order to solve the various design and application issues a huge amount of research is already carried out. Different continuous neighbour discovery schemes were proposed for efficient communication of a network and routing the fact data to the base station [9],[10][11][12][13][14].

DOI : $10.5121 /$ ijdps.2012.3409 
International Journal of Distributed and Parallel Systems (IJDPS) Vol.3, No.4, July 2012

In sensor networks Communication is totally depending upon the application. Hence, even after a sensor is aware of its immediate neighbours, it must continuously maintain its view; the sensors must continuously look for new neighbours in order to accommodate the following situations:

- Loss of local synchronization due to accumulated clock drifts;

- Disruption of wireless connectivity between adjacent nodes by a temporary event, such as a passing car or animal, a dust storm, rain, or fog; when these events are over, the hidden nodes must be rediscovered

- The on going addition of new nodes, in some network, to compensate for nodes that have ceased to function because their energy has been exhausted.

- The transmission power of some nodes to be increased, to certain actions, such as detection of emergency situations.

Because of the above reasons, for detecting the new links and nodes in sensor networks must be considered as continuous process. When the sensor networks performs continuous neighbour discovery, it is already responds of its immediate neighbours and can therefore perform it together with these neighbours in order to consume less energy. So, the initial neighbour discovery must be executed by each sensor independently.

In Fig; 1 shows a typical neighbour discovery protocol. In this process, a node becomes active according to its cycle. let this duty cycle be ${ }^{\alpha}$ in init state ${ }^{\beta}$ in normal state. From normal state to, we want to have ${ }^{\beta}<{ }^{\alpha}$. When a node becomes an active of its duty cycle; in this case it transmits a periodical HELLO messages and listens for similar message from possible neighbours. A node that receives messages means HELLO immediately responds and the remaining two nodes can invoke some other procedure to finalize the setup of the joint wireless link.

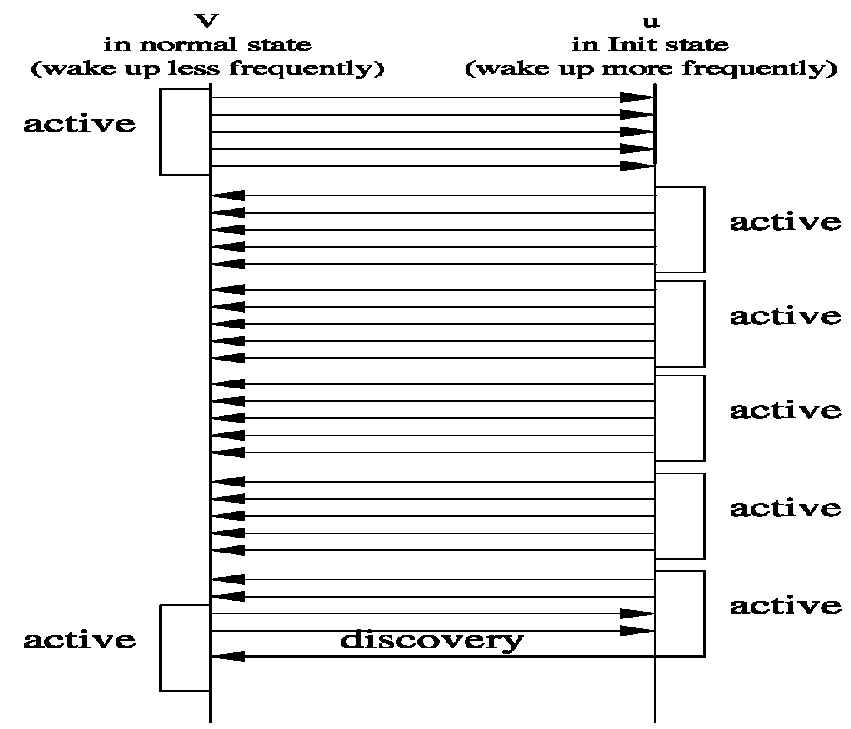

Fig. Transmission of HELLO messages in Init and Normal states

In the Initial state, in order to detect new neighbours, a node has no information about its surroundings and therefore must remain active for a relatively very long time. In the Normal state the node must use a more efficient scheme. This is the scheme, the subject of our study. 
International Journal of Distributed and Parallel Systems (IJDPS) Vol.3, No.4, July 2012

Fig. 2 It performs initial neighbour discovery, When node is in the Init state. After a certain time period, during which the node is expected, with high probability, to find most of its neighbours, the node moves to the Normal state, where continuous neighbour discovery is performed. A node in the Init state is also referred to as a hidden node, when the node in the normal state it can be referred as a segment node.

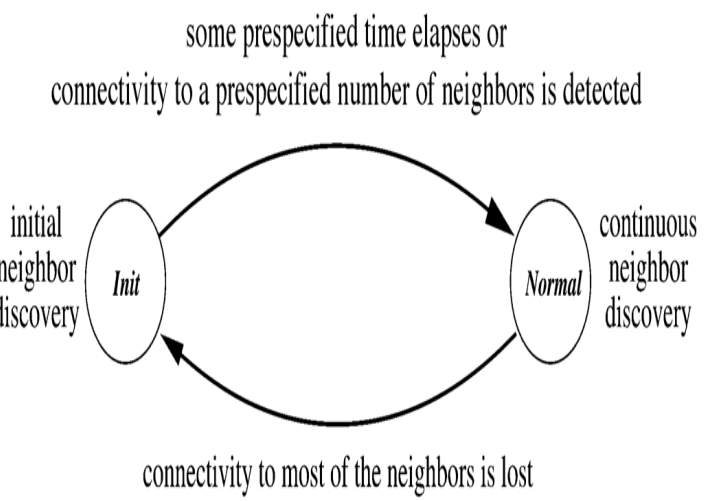

The main idea here, we propose is that the task of finding a new node $u$, is splitting among all the nodes that can help $\mathrm{v}$ to detect $\mathrm{u}$ nodes. These nodes are characterized as follows:

- They are also neighbors of $u$.

- They belong to a connected segment of nodes that have already detected each other

- Node $\mathrm{v}$ also belongs to this segment.

This variable indicates the in-segment degree of a hidden neighbour .In order to take advantage of the proposed discovery scheme node $v$ must estimate the value of degs $(u)$.

\section{Related work:-}

In a special node, called an access point, we are using this point in Wi-Fi network operating in centralized node. The Messages are transmitted only to or from the point. In the process of neighbour discovery, a new node can be detected by the base station. Discovering the new node is easy when compared the energy consumption is not a concern for the base station. The base station broadcasts a special HELLO message1. This message can hears that particular regular node to initiate a registration process. The regular node can switch frequencies/channels in order to handle the best HELLO message for its needs. This is the best message that might be depending on the identity of the broadcasting base station, on security considerations. All these Problems related the collisions of messages in such a network are addressed in [4]. So, other works trying to minimize the discovery time by optimizing the broadcast rate of the HELLO messages [1], [5], [6], [7], [8]. The main differences between neighbor discovery in WiFi and in mesh sensor networks are that neighbor discovery in the former are performed only by the central node, for which energy consumption is not a concern. The hidden nodes are assumed to be able to hear the HELLO messages; it can be broadcast by the central node. In the sensor networks is performed by every node, and hidden nodes cannot hear the HELLO messages when they sleep mode. In MANETs (Mobile Ad-hoc Networks), nodes usually do not switch to a special sleep state. Whenever their physical distance allows communication two neighbouring nodes can send messages to each other. AODV [9] is a typical routing protocol for MANETs. In 
International Journal of Distributed and Parallel Systems (IJDPS) Vol.3, No.4, July 2012

AODV, when a node wishes to send a message to another node, it broadcasts a special RREQ (route request) message.

For connectivity management the same message, of an established route maintenance procedure, aside from which there is no special neighbour discovery protocol. It is an important target design in Bluetooth [10]. As in Wi-Fi networks, this neighbour discovery in Bluetooth is also asymmetric. The 32 frequencies dedicated to neighbour discovery, the scan mode, and the node listens for a period on each while the discovering node passes through these frequencies one by one and broadcasts HELLO in each of them. A symmetric neighbour discovery scheme for Bluetooth is proposed in [11].

\section{PROBLEM DEFINITION:-}

We assume that all nodes are having the same transmission range, it means for every time the connectivity is always bi-directional. In our analysis, the network is a unit disk graph; means: the pair of the nodes that can be within transmission range are should be neighbouring nodes. These two nodes are said to be directly connected, and are aware of each other's wake-up times. Two nodes are said to be connected if there is a path of directly connected nodes between them. A group (set) of connected nodes is known as a segment. Consider a pair of neighbouring nodes that belong to the same segment but are not aware that they have Direct wireless connectivity.

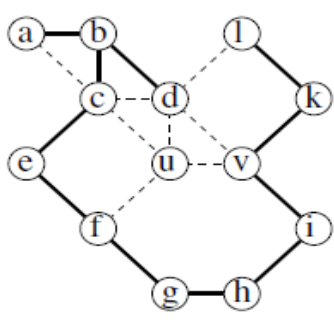

(a) a known link

a hidden link

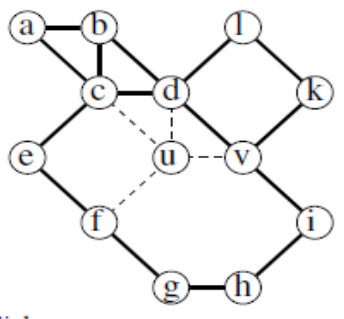

(b)

Fig. Segments with hidden nodes and links

In fig 3.(a) the nodes and c can learn about their hidden wireless link using the following simple scheme, which uses two message types:

(a) SYNC messages for synchronization between all segment nodes, transmitted over

Known wireless links;

(b) HELLO messages for detecting new neighbours.

\section{Scheme 1 (detecting all hidden links inside a segment):}

Whenever a new node is discovered by one of the segment nodes it can be detect the all hidden links inside a segment. For all segment members, the discovering node issues a special SYNC message asking them to periodically broadcast a group of HELLO messages. The SYNC 
message is passes over the already known wireless links of the segment. So, every segment node has to be guaranteed to be received.

\section{Scheme 2 (detecting a hidden link outside a segment):}

In this scheme, the same segment is used to minimize the possibility of repeating collisions between the HELLO messages of nodes. Practically, another scheme might be used, where segment nodes coordinate their wake-up periods for prevents collisions. However, finding an efficient time division is equivalent to the well-known node colouring problem, which is Node $\mathrm{u}$ wakes up randomly, every $\mathrm{T}(\mathrm{u})$ seconds on the

The value of $\mathrm{T}(\mathrm{u})$ is as follows:

- $\mathrm{T}(\mathrm{u})=\mathrm{TI}$, if node $\mathrm{u}$ is in the Init state

- $\quad \mathrm{T}(\mathrm{u})=\mathrm{TN}(\mathrm{u})$, if node $\mathrm{u}$ is in the Normal state

\section{Proposed method:-}

As already explained, we consider the discovery of hidden neighbours as a joint task to be performed by all segment nodes. We need to estimate the number of in-segment neighbours of every hidden node $\mathrm{u}$, denoted by $\operatorname{degS}(\mathrm{u})$ to determine the discovery load to be imposed on every segment node, namely, how often such a node should become active and send HELLO messages, In this section, I presents methods that can be used by node $\mathrm{v}$ in the Normal state to calculate this value. Node $\mathrm{u}$ is assumed to not yet be connected to the segment and it is in the Init (initial neighbour discovery) state. 1) here first we have to measures Node V, the average in-segment degree of the segment's nodes, we have to use this number as an estimate of the insegment degree of $u$. The average in-segment degree of the segment's nodes can be calculated by the segment leader. The end of this, it gets from every node in the segment and immediately a message indicating the in-segment degree of the sending node, which is known due to Scheme 1. 2) Node v discovers, using Scheme 1, the number of its in-segment neighbours, $\operatorname{degS}(\mathrm{v})$, and views this number as an estimate of $\operatorname{degS}(\mathrm{u})$. When the degrees of neighbouring nodes are strongly correlated, this approach will give good results than the previous one. 3) Node v uses the average in-segment degree of its segment's nodes and its own in-segment degree $\operatorname{degS}(\mathrm{v})$ to . To estimate the number of node u's neighbours. This approach gives the best results if the correlation between the in-segment degrees of neighbouring nodes is known.

\section{References}

[1] S. Vasudevan, J. Kurose, and D. Towsley, "On neighbor discovery in wireless networks with directional antennas," in INFOCOM, vol. 4, 2005, pp. 2502-2512.

[2] R. Madan and S. Lall, "An energy-optimal algorithm for neighbor discovery in wireless sensor networks," Mob. Netw. Appl., vol. 11, no. 3, pp. 317-326, 2006.

[3] M. J. McGlynn and S. A. Borbash, "Birthday protocols for low energy deployment and exible neighbor discovery in ad hoc wireless networks," in MobiHoc: Proceedings of the 2nd ACM International Symposium on Mobile Ad Hoc Networking and Computing. New York, NY, USA: ACM Press, 2001, pp. 137-145.

[4] D. Baker and A. Ephremides, "The architectural organization of a mobile radio network via a distributed algorithm," in IEEE Transactions on Communications, vol. 29, Nov. 1981, pp. 16941701.

[5] A. Keshavarzian and E. Uysal-Biyikoglu, "Energy-ef_cient link assessment in wireless sensor networks," in INFOCOM, 2004.

[6] E. B. Hamida, G. Chelius, and E. Fleury, "Revisiting neighbor discovery with interferences consideration," in PE-WASUN, 2006, pp. 74-81. 
International Journal of Distributed and Parallel Systems (IJDPS) Vol.3, No.4, July 2012

[7] S. A. Borbash, "Design considerations in wireless sensor networks," Ph.D. dissertation, ISR, August 2004.

[8] G. Alonso, E. Kranakis, R. Wattenhofer, and P. Widmayer, "Probabilistic protocols for node discovery in ad-hoc, single broadcast channel networks," in IPDPS, 2003, p. 218.

[9] C. Perkins, E. Belding-Royer, and S. Das, "Ad hoc on-demand distance vector (AODV) routing," RFC 3561, July 2003.

[10] J. Haartsen, Bluetooth Baseband Speci_cation v. 1.0.

[11] T. Salonidis, P. Bhagwat, L. Tassiulas, and R. O. LaMaire, "Distributed topology construction of bluetooth personal area networks," in INFOCOM, 2001, pp. 1577- 1586.

[12] IEEE 802.15.4: Wireless Medium Access Control (MAC) and Physical Layer (PHY) Speci_cations for Low-Rate Wireless Personal Area Networks (WPANs), IEEE 802.15 WPAN Task Group 4 (TG4), 2006.

[13] P. Dutta and D. Culler, "Practical asynchronous neighbor discovery and rendezvous for mobile sensing applications," in SenSys: Proceedings of the 6th ACM conference on Embedded network sensor systems. New York, NY: ACM Press, 2008, pp. 71-84.

[14] J. Hill and D. Culler, "A wireless embedded sensor architecture for system-level optimization," Technical report, U.C. Berkeley, 2001.

[15] R. Jurdak, P. Baldi, and C. V. Lopes, "Adaptive low power listening for wireless sensor networks," IEEE Transactions on Mobile Computing, vol. 6, no. 8, pp. 988-1004, 2007.

[16] Reuven Cohen and Boris Kapchits," Continuous Neighbor Discovery in synchronous Sensor Networks" IEEE Transactions on Mobile Computing, 2011.

[17] P. Dutta and D. Culler, .Practical asynchronous neighbor discovery and rendezvous for mobile sensing applications,. in SenSys: Proceedings of the 6th ACM conference on Embedded network sensor systems. New York, NY: ACM Press, 2008, pp. 71.84.

\section{Authors}

M.Sreedevi , MITS, Madanapalle , Received B.Tech in Electronics and communication Engineering from S.V.U.C.E, Tirupati, Andhra Pradesh, M.Tech Degree in Information Technology from Punjabi University , Patiala , Punjab and pursuing Ph.D in Cryptography and Network Security from S.V University, Tirupati, and 15years of professional experience.

Nadilla Khadar Valli, was born in Kadapa, October 12. He was received Bachelor's degree in Information technology in J.N.T University Hyderabad and M.Tech degree pursuing from J.N.T University Anantapur respectively. His research interest includes Computer Networks.

Dr.R.Seshadri, S.V.U, Tirupati, Received B.Tech Degree in Electronics \& communication Engineering from Nagarjuna university in 1981, Received M.E Degree from PSG College of technology, Coimbatore in 1984, Received Ph.D in Simulation modeling and compression of ECG signals from S.V.UNIVERSITY. He had 30 years of Professional experience.
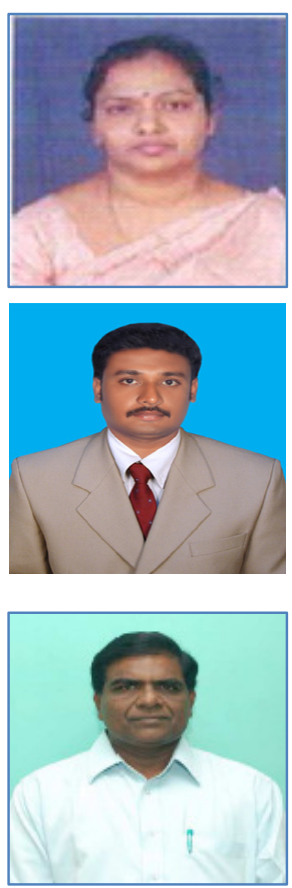